\section{Tratamento do Tumor do Córtex Adrenal na Infância}

\section{RESUMO}

O tumor do córtex adrenal (TCA) na infância é raro. Entretanto, no Paraná sua incidência é 15 vezes maior do que a observada em outros países. A mutação germinativa R337H TP53, presente em mais de $95 \%$ dos nossos pacientes, provavelmente está relacionada à maior incidência. Cento e vinte e cinco pacientes foram tratados no período de 1966 a 2003. A cirurgia é o único tratamento curativo. Em nossa experiência, tumores no estádio I, ausência de spillage durante a cirurgia e ausência de trombo são parâmetros relacionados à maior sobrevida. Dados preliminares mostram que a associação de etoposídeo, doxorrubicina, cisplatina e mitotano produziu remissão completa do tumor e/ou das metástases em alguns pacientes. Os efeitos colaterais destas drogas são comuns e pode ocorrer insuficiência adrenocortical. As doses de reposição de glicocorticóides e mineralocorticóides devem ser 2 a 3 vezes maiores que as doses fisiológicas. (Arq Bras Endocrinol Metab 2005;49/5:747-752)

Descritores: Tumor de córtex adrenal; Infância

\begin{abstract}
Treatment of Childhood Adrenocortical Tumor.

Adrenocortical tumors (ACT) in children are uncommon. However, the incidence of these tumors in Paraná is 15 times higher than that worldwide. A germline mutation, R337H TP53, present in more than $95 \%$ of our patients, is probably the reason for the higher incidence in our state. A hundred twenty-five patients were treated in the period of 1966 to 2003. Surgery is the only curative treatment. In our experience, disease stage I, absence of spillage during surgery and absence of intravenous thrombus are associated with better survival rates. Preliminary data with the combination of etoposide, doxorubicin, cisplatin, and mitotane have shown that in some patients a complete remission is observed both of the tumor and metastasis. Side effects due to these drugs are common and adrenal insufficiency may occur. Glucocorticoid and mineralocorticoid reposition should be done with 2 to 3 times the physiological doses. (Arq Bras Endocrinol Metab 2005;49/5:747-752)
\end{abstract}

Keywords: Adrenocortical tumor; Children

$\mathrm{O}$ TUMOR DO CÓRTEX ADRENAL (TCA) é raro; corresponde a aproximadamente $0,2 \%$ dos casos de câncer na infância, com incidência variável de acordo com a área geográfica (1). A maioria dos registros com base em dados populacionais reporta uma prevalência de 0,5 casos/ 1.000 .000 (2). Apesar da sua raridade, nas regiões Sul e Sudeste do Brasil a incidência do TCA é de 3,4-4,2 casos/1.000.000 (3), aproximadamente 15 vezes maior que a incidência mundial. Dados recentes, baseados na taxa revisão

Rosana Marques Pereiva Edson Michalkiewicz Mara Albonei Pianovski Susana Nesi França Margaret C.S. Boguszewski Izrail Cat Luiz de Lacerda Filbo Romolo Sandrini

Unidade de Endocrinologia Pediátrica (RMP, MCSB, LLF \& RS), Serviço de Hemato-Oncologia Pediátrica (MAP) e Unidade de Terapia Intensiva (IC) do Departamento de Pediatria, Hospital de Clinicas,

Universidade Federal do Paraná; Serviço de Cirurgia Oncológica Pediátrica, Hospital Erasto Gaertner (EM), Curitiba, PR.

Recebido em 18/08/05

Revisado em 27/08/05 Aceito em 01/09/05 
de mortalidade por TCA em Curitiba e região metropolitana, confirmam esta incidência (Pianovski e cols., 2005, submetido). Esta diferença está relacionada à presença da mutação germinativa R337H TP53. Esta mutação, localizada no domínio de tetramerização da proteína, é o único exemplo de mutação no gene TP53 relacionada a apenas um câncer (4).

$\mathrm{Na}$ Unidade de Endocrinologia Pediátrica (UEP) do Hospital de Clínicas da Universidade Federal do Paraná, são diagnosticados 6 a 8 casos novos/ ano; entre 1966 e 2003 foram atendidas 125 crianças portadoras de TCA. O diagnóstico histológico do tumor, que em muitos casos de câncer determina a conduta terapêutica e o prognóstico, não tem o mesmo valor nos casos de TCA. Nestes tumores nem sempre é possível a diferenciação entre adenoma e adenocarcinoma (5). Entretanto, na nossa experiência nenhum paciente que teve um tumor com diagnóstico anátomo-patológico de adenoma apresentou recidiva da doença.

O estadiamento do TCA na infância (tabela 1 ), a presença de trombo intravenoso, a presença ou não de spillage (ruptura da cápsula do tumor durante a cirurgia ou exposição de trombo vascular tumoral durante a cirurgia) e a retirada completa do tumor e dos trombos são parâmetros importantes para 0 prognóstico (6).

O tratamento do TCA requer uma equipe multidisciplinar constituída, entre outros, do endocri-

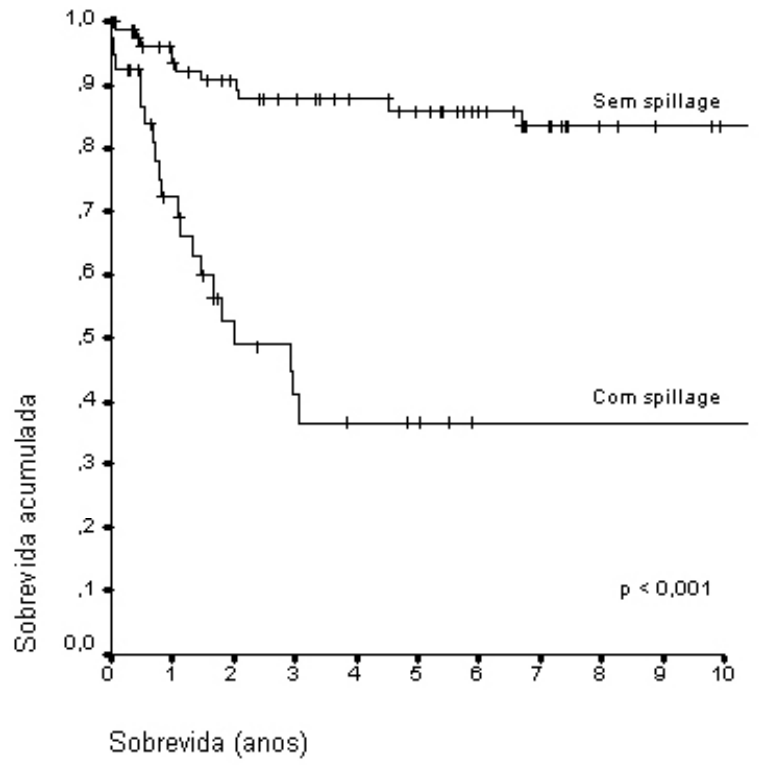

Figura 1. Gráfico de Kaplan-Meier, com a sobrevida conforme a presença ou não de spillage, em 125 pacientes. nologista pediátrico, do cirurgião pediátrico oncológico, do oncologista pediátrico, do intensivista e, eventualmente, do cirurgião cardiovascular. O endocrinologista pediátrico deve seguir estes pacientes segundo um protocolo, em virtude dos vários eventos relacionados ao tumor (hipertensão, puberdade precoce, crescimento e estatura final) ou ao tratamento (insuficiência adrenal, por exemplo). Diferente do que acontece nos casos de TCA nos adultos, a maioria dos TCA na infância são funcionantes, sendo a virilização isolada ou associada à síndrome de Cushing as principais formas de apresentação clínica $(6,7)$.

Pacientes que apresentam doença nos estádios I e II são apenas submetidos à cirurgia. Pacientes com doença no estádio III e IV são submetidos à cirurgia e quimioterapia.

\section{CIRURGIA}

A terapêutica com maior índice de cura para o TCA na infância é a ressecção completa do tumor, sem ruptura da cápsula tumoral, especialmente nos tumores nos estádios I e II (6). A laparotomia transperitoneal é a via de acesso mais adequada para ressecção completa do tumor, dos linfonodos retroperitoneais regionais e inventário da cavidade abdominal (8). A ruptura da cápsula do tumor, com extravasamento de células neoplásicas no leito cirúrgico ou na cavidade abdominal (spillage), pode ocorrer antes ou durante a cirurgia e aumenta consideravelmente o risco de recidiva local (9) e diminui significativamente a sobrevida (figura 1). A ruptura pré-operatória é rara (10) e pode ocorrer durante a cirurgia devido a dificuldades técnicas (tumores por vezes grandes e friáveis).

Cento e vinte e três pacientes foram submetidos à cirurgia curativa ou diagnóstica. A grande maioria dos pacientes $(86,2 \%)$ teve o tumor completamente ressecado; 3,3\% tiveram ressecção parcial (tumor residual macroscópico) e somente biópsia foi realizada em 10,6\% dos pacientes (estes na década de 60).

Tabela 1. Estadiamento do TCA em crianças.

\begin{tabular}{ll}
\hline Estádio & Descrição \\
\hline I & $\begin{array}{l}\text { Tumor totalmente ressecado; peso do tumor } \\
\text { 200g; ausência de metástase. }\end{array}$ \\
II & $\begin{array}{l}\text { Tumor totalmente ressecado com margens } \\
\text { cirúrgicas negativas; peso do tumor > 200g; } \\
\text { ausência de metástase. }\end{array}$ \\
III $\quad \begin{array}{l}\text { Tumor residual (micro ou macroscópico) ou } \\
\text { inoperável, sem metástase. }\end{array}$ \\
IV $\quad$ Metástase a distância no diagnóstico.
\end{tabular}

Arq Bras Endocrinol Metab vol $49 n^{\circ} 5$ Outubro 2005 
A experiência atualmente acumulada com a adrenalectomia endoscópica para tumores benignos em adultos não deve ser estendida aos TCA da infância e adolescência, pela falta de elementos que permitam o diagnóstico de doença benigna e, especialmente, pela possibilidade de ruptura de cápsula, recidiva local e disseminação em casos de doença maligna (11-13).

Trombo tumoral intravenoso foi encontrado em 19,6\% de 92 pacientes investigados, principalmente nas veias supra-renal, renal, cava e no átrio direito. Uma vez constatada a presença do trombo, deve-se ressecá-lo juntamente com o tumor primário. Quando ele é extenso e atinge o coração, torna-se necessária a intervenção do cirurgião cardiovascular e utilização de circulação extracorpórea, a qual facilita a ressecção completa do trombo.

Em doença metastática ao diagnóstico ou em recidiva, local ou com metástase, deve-se tentar a ressecção completa seguindo os mesmos princípios indicados para a ressecção do tumor primário (14). Esta conduta tem curado ou prolongado a sobrevida de alguns pacientes. Um deles está sem a doença há 20 anos, após 2 cirurgias para retirada de recidiva local e uma para hemi-hepatectomia por metástase hepática. Outro paciente, com diagnóstico aos 9 meses de idade, apresentou 10 recidivas locais e à distância e foi operada em cada recidiva, tendo sobrevivido por cinco anos.

Todos os pacientes recebem hidrocortisona imediatamente antes, durante e após a adrenalectomia.

A maioria dos pacientes sem ressecção completa do tumor morre entre 3 e 12 meses.

\section{HIPERTENSĀO}

Hipertensão arterial foi observada, por ocasião do diagnóstico, em $56 \%$ dos 125 pacientes avaliados no nosso serviço e esteve presente em todas as formas

Tabela 2. Características clínicas e terapêuticas de 4 crianças sem doença mensurávela submetidas à quimioterapia.

\begin{tabular}{|c|c|c|c|c|}
\hline \multicolumn{5}{|c|}{ Paciente Idade (meses) Sexo Indicaçāo Evoluçāo } \\
\hline AK & 52 & $\mathrm{~F}$ & MP & Remissãob \\
\hline MSA & 72 & M & RL & Recidiva \\
\hline $\begin{array}{l}\text { NMS } \\
\text { da }\end{array}$ & 130 & $\mathrm{~F}$ & MP & $\begin{array}{l}\text { Remissão e posterior recidiva } \\
13 \text { meses após término } \\
\text { quimioterapia }\end{array}$ \\
\hline $\begin{array}{l}\text { DPS } \\
\text { da }\end{array}$ & 168 & M & MP & $\begin{array}{l}\text { Remissão e posterior recidiva } \\
18 \text { meses após término } \\
\text { quimioterapia }\end{array}$ \\
\hline
\end{tabular}

a Com ressecção completa de recidiva e/ou metástase

b Paciente faleceu por provável insuficiência supra-renal clínicas do TCA. Nove pacientes apresentaram encefalopatia hipertensiva, com crise convulsiva. A hipertensão pode persistir por dias ou semanas após a ressecção do tumor (6). A hipertensão ocorreu em $13,6 \%$ dos pacientes com quadro clínico e laboratorial de virilização isolada. Sua patogênese pode estar relacionada à compressão da artéria renal pelo tumor (9) ou à secreção de um ou mais esteróides não investigados na nossa casuística (15). No manejo da hipertensão, em geral há boa resposta à terapêutica com inibidores da ECA, utilizados em esquema de monoterapia ou em associação com diurético ou com cetoconazol nas formas graves (6).

\section{QUIMIOTERAPIA}

A quimioterapia tem acrescentado pouco impacto à chance de cura. Contudo, nos casos em que a ressecção completa do tumor não é possível e nos tumores metastáticos, o tratamento quimioterápico adjuvante, embora controverso, tem sido indicado $(3,16,17)$.

Em 1998, Berruti e cols. (18) descreveram 2 pacientes com resposta completa e 13 com resposta parcial, taxa de resposta global de $53,5 \%$, após o tratamento com a combinação de cisplatina, doxorrubicina, etoposídeo e mitotano. Estes quimioterápicos são habitualmente usados no tratamento do TCA, de forma isolada ou associados. A vantagem da administração concomitante com o mitotano é a possível capacidade que ele apresenta em potencializar os agentes quimioterápicos (18). Além do seu efeito adrenolítico, estudos in vitro mostram que o mitotano tem a capacidade de reverter a resistência de linhagens de células resistentes a múltiplas drogas. Estas células expressam o gene MDR-I (multidrug resistance 1 gene), presente também em células de TCA. É possível que a falha nos esquemas quimio-

Tabela 3. Características clínicas e terapêuticas de 6 crianças com doença mensurável submetidas à quimioterapia.

\begin{tabular}{lcccc}
\hline $\begin{array}{l}\text { Paciente } \\
\text { atingir }\end{array}$ & Idade (meses) Sexo & Indicação & $\begin{array}{c}\text { Tempo para } \\
\text { remissão (meses) }\end{array}$ \\
\hline ABS & 25 & $\mathrm{M}$ & $\mathrm{R}+\mathrm{MH}$ & 4 \\
GCS & 39 & $\mathrm{~F}$ & $\mathrm{MP}$ & 5 \\
SVG & 73 & $\mathrm{~F}$ & $\mathrm{MP}$ & 4 \\
KDS & 66 & $\mathrm{~F}$ & $\mathrm{MP}$ & 4 \\
JF & 180 & $\mathrm{~F}$ & $\mathrm{R}+\mathrm{MP}$ & Nãoa \\
GMS & 20 & $\mathrm{M}$ & Tumor Irressecável & 2 \\
\hline a Paciente não apresentou remissão da doença. Houve
\end{tabular}
resposta parcial e está em tratamento há 18 meses. 
terápicos utilizados para o tratamento de TCA esteja ligada à presença do $M D R-1$ (19).

A dose do mitotano proposta por Berruti e cols. foi por nós modificada ( $4 \mathrm{~g} /$ dia para $4 \mathrm{~g} / \mathrm{m}^{2} /$ dia). Estamos utilizando este esquema para pacientes em estádio III ou IV, e em recidivas para pacientes que apresentavam estádio I ou II inicialmente.

Para acelerar o depósito de mitotano no tecido adiposo, o mesmo é misturado com triglicerídeos de cadeia média e outros alimentos gordurosos rotineiramente ingeridos na dieta do paciente (leite, chocolate, iogurte). Os efeitos colaterais são intensos e freqüentes, incluindo lesão temporária ou permanente da adrenal contralateral, ginecomastia, artralgia, rash, náuseas, vômitos, diarréia e sintomas neurológicos como letargia, sonolência, mudança do humor e, mais raramente, coma $(19,20)$. É freqüente a falta de adesão por intolerância gástrica. Para alguns pacientes, temos indicado o uso de sonda naso-enteral para garantir a administração da droga.

O nível sérico de mitotano deve ser monitorizado, com determinações freqüentes, com objetivo de manutenção do nível terapêutico e não-tóxico, situado entre 14 e $20 \mathrm{mcg} / \mathrm{mL}(18,22,23)$. Cuidado especial deve ser dado à interpretação de náusea e vômito que, embora comumente associados à quimioterapia, podem ser sinais de insuficiência adrenal.

Além dos efeitos colaterais do mitotano citados anteriormente, outros efeitos da droga relacionam-se ao funcionamento da glândula adrenal normal e ao metabolismo dos corticoesteróides administrados ao paciente. Em doses $\leq 3 \mathrm{~g} /$ dia, o mitotano induz à supressão da esteroidogênese adrenal; em doses > 3g/dia, é observado um efeito adrenolítico (16). A reposição de glicocorticóide e mineralocorticóide deve ser instituída até duas semanas após

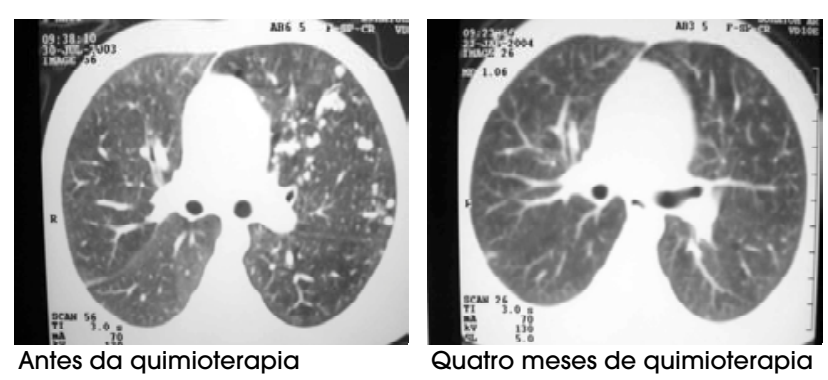

Figura 2. Tomografia axial computadorizada de tórax mostrando remissão completa de metástases pulmonares de TCA por quimioterapia com etoposídeo, doxorrubicina, cisplatina e mitotano, em uma menina de 5 anos de idade. o início do mitotano (16). Em virtude dos efeitos do mitotano sobre o metabolismo dos glicocorticóides e dos mineralocorticóides, as doses de reposição devem ser maiores que aquelas consideradas fisiológicas $(16,20,24)$. Robinson e cols. demonstraram aumento do clearance metabólico da dexametasona nos pacientes em uso do mitotano (20). Utilizamos prednisona ( 10 a $15 \mathrm{mg} / \mathrm{m}^{2} / \mathrm{dia}$ ), dividida em 2 a 3 tomadas, ou dexametasona $\left(2 \mathrm{a} 3 \mathrm{mg} / \mathrm{m}^{2} / \mathrm{dia}\right)$ em 2 tomadas, e fluodrocortisona, 0,15 a $0,2 \mathrm{mg} / \mathrm{dia}$. Em situações de estresse, a dose do glicocorticóide deverá ser triplicada, retornando-se à dose habitual após a resolução do fator de estresse.

O mitotano deposita-se no tecido celular subcutâneo, fígado, cérebro e adrenais e sua concentração sérica permanece elevada até meses após a suspensão do seu uso (20). Portanto, a necessidade de reposição de corticoesteróides persiste mesmo após a droga ter sido descontinuada (20-22). Avaliação clínica cuidadosa e as concentrações séricas de sódio, potássio, glicose e ACTH são parâmetros importantes para a adequação das doses de reposição dos glico e mineralocorticóides.

Não há consenso quanto ao tempo de tratamento com mitotano $(16,18,25)$, mas nos pacientes que estão em remissão deve ser mantido por um período mínimo de 18 meses. A quimioterapia é descontinuada nos pacientes que apresentam recidiva durante o tratamento.

Utilizamos o esquema de Berruti e cols. (18) modificado, em 8 pacientes. Resultados preliminares demonstram que é possível conseguir remissão completa e prolongada de lesões metastáticas e/ou pulmonares (tabelas 2 e 3, figura 2). Cinco pacientes apresentaram remissão completa, por períodos que variaram de 6 a 20 meses.

\section{CONCLUSÕES}

A cirurgia é a modalidade terapêutica mais efetiva do TCA na infância, especialmente nos tumores estádio I e II. A ocorrência de spillage durante a cirurgia diminui significativamente a sobrevida dos pacientes. Resultados preliminares com a quimioterapia composta por cisplatina, etoposídeo, doxorrubicina e mitotano mostram que é possível obter remissão completa do tumor e/ou metástases. Efeitos colaterais importantes são observados com este esquema terapêtico, relacionados às drogas ou à insuficiência adrenocortical. Doses de reposição de glicocorticóides e mineralocorticóides devem ser 2 a 3 vezes as doses consi- 
deradas fisiológicas.

\section{REFERÊNCIAS}

1. Figueiredo B, Ribeiro RC. Childhood adrenocortical tumours. Eur J Cancer 2004;40:1117-26.

2. Parkin DM, Stiller CA, Draper GJ, Bieber CA. The international incidence of childhood cancer. In J Cancer 1988;42:511-20.

3. Sandrini R, Ribeiro RC, De Lacerda L. Childhood adrenocortical tumors. J Clin Endocrinol Metab 1997;82:2027-31.

4. Gallo CVM, Azevedo G, Mendonça S, et al. TP53 mutations as biomarkers for cancer epidemiology in Latin America: current knowledge and perspectives. Mutat Res 2005;589:192-207.

5. Bugg MF, Ribeiro RC, Roberson PK, Lloyd RV, Sandrini R, Silva JB, et al. Correlation of pathologic features with clinical outcome in pediatric adrenocortical neoplasia. Am J Clin Pathol 1994;101:625-9.

6. Pereira RM, Michalkiewicz E, Sandrini F, Figueiredo BC, Pianovski M, Franca SN, et al. Tumores do córtex adrenal na infância. Arq Bras Endocrinol Metab 2004;48/5:651-8.

7. Latronico AC, Chrousus GP. Adrenocortical tumors. J Clin Endocrinol Metab 1997;82:1317-24.

8. Nagesser SK, Kievit J, Hermans J, Krans HM, Van V. The surgical approach to the adrenal gland: a comparison of the retroperitoneal and the transabdominal routes in 326 operations on 284 patients. Jpn J Clin Oncol 2000;30:68-74.

9. Michalkiewicz E, Sandrini R, Figueiredo B, Miranda EC, Caran E, Oliveira-Filho AG, et al. Clinical and outcome characteristics of children with adrenocortical tumors: A report from the International Pediatric Adrenocortical Tumor Registry. J Clin Oncol 2004;5:838-45.

10. Lack EE, Mulvihill JJ, Travis WD, Kozakewich HOW. Adrenocortical neoplasm in the pediatric and adolescent age group: Clinicopathologic study of 30 cases with emphasis on epidemiological and prognostic factors. Pathol Ann 1992;27:1-53.

11. Deckers S, Derdelinckx L, Col V, Hamels J, Maiter D. Peritoneal carcinomatosis following laparoscopic resection of an adrenocortical tumor causing primary hyperaldosteronism. Horm Res 1999;52:97-100.

12. Foxius A, Ramboux A, Lefevre Y, Broze B, Hamels J, Squifflet J. Hazards of laparoscopic adrenalectomy for Conn's adenoma. When enthusiasm turns to tragedy. Surg Endosc 1999;13:715-7.

13. lino K, Oki Y, Sasano H. A case of adrenocortical carcinoma associated with recurrence after laparoscopic surgery. Clin Endocrinol (Oxf) 2000;53:243-8.

14. Pommier RF, Brennan MF. An eleven-year experience with adrenocortical carcinoma. Surgery 1992;112:96370; discussion 970-1.

15. Kater CE, Biglieri EG, Brust N, Chang B, Hirai J, Irony I. Stimulation and suppression of the mineralocorticoid hormones in normal subjects and adrenocortical disorders. Endocr Rev 1989;10:149-64.
16. Wajchenberg BL, Pereira MAA, Mendonça BB, Latronico AC, Campos Carneiro P, Alves VA, et al. Adrenocortical carcinoma. Clinical and laboratory observations. Cancer 2000;88:711-36.

17. Chudler RM, Kay R. Adrenocortical carcinoma in children. Urol Clin North Am 1989;16:469-79.

18. Berruti A, Terzolo M, Pia A, Angeli A, Dogliotti L, for the Italian Group for the study of adrenal cancer. Mitotane associated with etoposide, doxorrubicin and cisplatin in the treatment of advanced adrenocortical carcinoma. Cancer 1998;83:2194-200.

19. Bates SE, Shieh C, Mickley LA, Dichek HL, Gazdar A, Loriaux DL, et al. Mitotane enhances cytotoxicity of chemotherapy in cell lines expressing a multidrug resistance gene (mdr-1/P-glycoprotein) which is also expressed by adrenocortical carcinomas. J Clin Endocrinol Metab 1991;73:18-29.

20. Robinson BG, Hales IB, Henniker AJ, Ho K, Luttrell BM, Smee IR, et al. The effect of o-p'DDD on adrenal steroid replacement therapy requirements. Clin Endocrinol 1987;27:437-44

21. Hogan TF, Citrin DL, Johnson BM, Nakamura S, Davis TE, Borden EC. o,p'-DDD (mitotane) therapy of adrenal cortical carcinoma: observations on drug dosage, toxicity, and steroid replacement. Cancer 1978;42:2177-81.

22. Andersen A, Kasoerkiki-Zaluska AA, Warren DJ. Determination of mitotane (o-p'-DDD) and its metabolites op'-DDA and o-p'-DDE in plasma by high- performance liquid chromatography. Ther Drug Monit 1999;21:33540.

23. Andersen A, Warren DJ, Nome O, Vesterhus L, Slordal L. A high-pressure liquid chromatography method for measuring mitotane (1,1-(o-p'-dichlorodiphenyl)-2-2dichloroethane) and its metabolite 1,1-(o-pl'-Dichlorodiphenyl) -2-2-dichloroethene in plasma. Ther Drug Monit 1995;17:526-31.

24. Baudin E, Pellegriti G, Bonnay M, Penfornis A, Laplanche $A$, Vassal $G$, et al. Impact of monitoring plasma 1,1dichlorodiphenilcichloroethane (o,p'DDD) levels on the treatment of patients with adrenocortical carcinoma. Cancer 2001:92:1385-92.

25. Orth DN, Kovacs WJ, Debold CR. The Adrenal Cortex. In: Wilson \& Foster, editors. Williams Textbook of Endocrinology. $8^{\text {th }}$ ed. Philadelphia:WB Saunders, 1992 p.489-619.

26. Young JL, Miller RW. Incidence of malignant tumors in US children. J Pediatr 1975;86:254-8.

27. Wieneck JA, Thompson LDR, Heffess C. Adrenal cortical neoplasm in the pediatric population. Am J Surg Pathol 2003;27:867-81.

28. Koch CA, Pacak K, Chrousos GP. The molecular pathogenesis of hereditary and sporadic adrenocortical and adrenomedullary tumors. J Clin Endocrinol Metab 2002;87:5367-84.

29. Ribeiro RC, Sandrini F, Figueiredo B, Zambetti GP, Michalkiewicz $E$, Lafferty $A R$, et al. An inherited p53 mutation that contributes in a tissue-specific manner to pediatric adrenal cortical carcinoma. Proc Nat Acad Sci USA 2001;98:9330-5.

30. Latronico AC, Pinto EM, Domenice S, Fragoso MC, 
Martin RM, Zerbini MC, et al. An inherited mutation outside the highly conserved DNA-binding domain of the p53 tumor supressor protein in children and adults with sporadic adrenocortical tumors. J Clin Endocrinol Metab 2001;86:4970-3.

31. Figueiredo $B$, Ribeiro $R$, Ellen $M$, et al. Multiple childhood adrenocortical tumors in six families from Southern Brazil without Li-Fraumeni syndrome. Horm Res 2000;53(suppl. 2):15.

32. Lefevre M, Gerard-Marchant R, Gubler JP, Chaussain JL, Lemerle J. Adrenal cortical carcinoma in 42 patients treated from 1958 to 1980 at Villejuif. In: Humphrey GB, Grindey GB Dehner LB, editors. Adrenal and endocrine tumors in children. Boston:Martinus Nijhoff Publishers, 1984.p.256-76.

33. Kersjes W, Mayer E, Buchenorth M, Schunk K, Fouda N, Cagil H. Diagnosis of pulmonary metastases with turboSE MR imaging. Eur Radiol 1997;7:1 190-4.

34. Pianovski MAD, Lacerda Filho L, Figueiredo $B C$, et al. ICE (Ifosfamide, Carboplatin and Etoposide) combination chemotherapy in the treatment of children with advanced adrenocortical carcinoma. Med Ped Oncol 1999;33:279.
35. Haak HR, Hermans J, Van de Velde CJ, Lentjes EG, Goslings BM, Fleuren GJ, et al. Optimal treatment of adrenocortical carcinoma with mitotane: results in a consecutive series of 96 patients. Br J Cancer 1994:69:947-51.

36. Patil KK, Ransley PG, McCullagh M, Malone M, Spitz L. Functioning adrenocortical neoplasms in children. BJU International 2002;89:562-5.

\section{Endereço para correspondência:}

Romolo Sandrini

Unidade de Endocrinologia Pediátrica

Departamento de Pediatria, Hospital de Clínicas

R. Padre Camargo 250

80060-240 Curitiba, PR

E-mail: sandrini@ufpr.br 